\title{
Predictors of pulmonary function at six years of age in infants with bronchopulmonary dysplasia
}

\author{
Brianna Aoyama ${ }^{1}$, Joseph Collaco ${ }^{2}$, and Sharon McGrath-Morrow ${ }^{3}$ \\ ${ }^{1}$ Johns Hopkins Medical Institutions Campus \\ ${ }^{2}$ Johns Hopkins Medical Institutions \\ ${ }^{3}$ Children's Hospital of Philadelphia
}

October 12,2020

\begin{abstract}
Rationale: Bronchopulmonary dysplasia (BPD) is a major complication of premature birth and the most common cause of chronic lung disease in infancy. Previous studies have shown that children with a history of BPD have impaired lung function in childhood compared to their term counterparts. However, little is known about potential modifiable factors that alter lung function trajectories and subsequent respiratory morbidity in this population. Objectives: To identify potential modifiable risk factors for the development of impaired lung function in patients with a history of prematurity and BPD. Methods: Growth parameters (birth, 2yo, 6yo) and pulmonary function testing (6yo) were retrospectively reviewed for subjects ( $\mathrm{n}=598)$ recruited from an outpatient BPD clinic who were born [?]36 weeks gestation and were [?]5 years of age. Results: Of the 598 recruited subjects, $88(14.7 \%)$ performed adequate pulmonary function testing at approximately six years of age. The mean FEV1\% predicted was $84.5 \%$ with lower values associated with lower median household income, Nissen fundoplication, and higher weight percentiles at 2 yo. The mean FVC $\%$ predicted was $94.2 \%$ with lower values associated with higher amounts of oxygen required at time of initial hospital discharge, Nissen fundoplication, and higher weight percentiles at 2yo. Conclusions: Our study found that children with BPD have different long-term pulmonary trajectories than full-term controls. Supplemental oxygen, lower income, and Nissen fundoplication at discharge were associated with lower lung function at 6 years of age. Prospective studies should focus on modifiable risk factors that could minimize the impact of BPD on later lung function.
\end{abstract}

\section{INTRODUCTION}

Preterm birth, defined as birth at less than thirty-seven weeks gestation, is the leading cause of mortality in children under five years of age and an important cause of morbidity, associated with prolonged hospitalizations and multisystem complications that persist throughout childhood and into adulthood ${ }^{1}$. Rates of preterm birth have increased globally over the past twenty years and currently preterm births account for more than $11 \%$ of livebirths ${ }^{2-3}$. Bronchopulmonary dysplasia (BPD) is a major complication of preterm birth and the most common cause of chronic lung disease in infancy. Based on the 2001 National Heart, Lung, and Blood Institute (NHLBI) definition ${ }^{4}$, it is estimated that up to 50,000 infants develop BPD each year in the United States ${ }^{5-6}$. While advances in neonatal care and technology, such as gentler ventilation strategies, exogenous surfactant, and the widespread use of postnatal steroids, have led to improved survival and outcomes for these infants, the incidence of BPD, which is defined as the need for supplemental oxygen for at least twenty-eight days after birth, has remained unchanged ${ }^{6}$.

Several previous studies, including a meta-analysis by Kotecha et al. ${ }^{7}$, have shown that preterm patients, and especially those with BPD, display significant lung function impairment later in life compared to their term peers. These individuals are at an increased risk of respiratory morbidity later in childhood, with increased respiratory symptoms, structural abnormalities, and decreased lung function, primarily characterized by airway obstruction, altered peripheral lung mechanics, and hyperinflation ${ }^{8}$. A study of two hundred 
preterm children in Australia, including 126 with BPD and 74 without BPD, demonstrated that lung function trajectories in survivors of preterm birth are impaired and those with BPD, ongoing respiratory symptoms, or findings on chest computed tomography (CT) have the poorest trajectories, and are at the greatest risk of developing significant lung disease in adulthood ${ }^{8}$. Similar results were appreciated in a longitudinal observational study of the EPICure cohort ${ }^{9}$, a cohort of extremely low birth weight infants which showed that individuals displayed impaired lung function and increased respiratory morbidity that persisted into middle childhood, defined as eleven years of age, with findings especially notable in infants with BPD. Interestingly, this impairment was appreciated in infants born in both the pre-surfactant era as well as the modern era during which new management techniques have been employed ${ }^{10-11}$. These findings all suggest that longterm follow-up is especially important in this population given the concern for chronic obstructive pulmonary disease during adult life. This population has remained steady and even grown in size in the past several years ${ }^{12}$, and as a result, the global burden of lung disease following preterm birth is set to rise which has significant implications for adult providers and the health care system as a whole.

Overall, however, little is known about the potential modifiable predictors of lung function in this population specifically during the first two years of life, a period of postnatal life in which alveolar growth occurs most rapidly ${ }^{13}$. One retrospective study of 322 preterm infants with BPD showed that the initial severity of an individual's BPD was an important predictor of pulmonary function abnormalities (at eight years old and then subsequently at fifteen years old) as well as health care usage during childhood ${ }^{14}$. The aim of this study was to investigate demographic and clinical characteristics as well as growth measurements at birth and at two years of age to determine what factors are most strongly predictive of lung function at six years of age. We hypothesized that in the outpatient setting, socioeconomic factors, growth parameters, and need for respiratory support would influence lung function at 6 years of age in children with a history of bronchopulmonary dysplasia.

\section{METHODS}

\section{Population and Study Design:}

A retrospective chart review was performed using patients $(\mathrm{n}=598)$ who were recruited from an outpatient BPD clinic between January 2008 and February 2020 and were at least five years of age as of February 2020. All subjects were born at less than or equal to 36 weeks gestation and presented with respiratory symptoms. BPD severity was defined by the 2001 NHLBI workshop definition ${ }^{4}$. While at the time of enrollment in the study, all subjects were outpatients at a single center, they received their neonatal care at a variety of neonatal intensive care units (NICUs) across the state prior to referral to the outpatient BPD clinic for follow-up. All caregivers were consented per study protocol as approved by the Johns Hopkins University Institutional Review Board (Protocol NA_00051884).

\section{Clinical Data :}

Clinical data were obtained through chart review for birth parameters, clinical characteristics, growth parameters at both two and six years of age, and pulmonary function testing data obtained at six years of age. Bronchopulmonary dysplasia was defined as the need for oxygen supplementation for twenty-eight days of life and a "physiologic" assessment of the oxygen requirement at thirty-six weeks corrected age for determining severity $^{4}$. Percentile for birth weight was corrected for gestational age using national norms ${ }^{15}$, and later height, weight, and body mass index measurements were expressed as quantitative data and percentile-forage measurements based on CDC norms.

\section{Pulmonary Function Testing :}

Patients performed spirometry at approximately six years of age at a routine clinic visit when well and without symptoms of an acute respiratory tract infection. Testing was performed using MedGraphics Platinum Elite Series Plethysmograph at two different clinic locations and according to the American Thoracic Society (ATS) and European Respiratory Society (ERS) standards ${ }^{16}$. Patients were adequately instructed and trained before proceeding with testing. Forced vital capacity (FVC) and forced expiratory volume in 1 second 
$\left(\mathrm{FEV}_{1}\right)$ were recorded and adjusted for age, height, and race per protocol. Quantitative measurements as well as percent predicted values were recorded ${ }^{17}$. Spirometry measurements were accepted if they met the acceptability and repeatability criteria of the ATS and ERS ${ }^{16}$. All spirometry performed and used in analysis was interpreted by an attending board-certified pediatric pulmonologist.

\section{Statistical Methods :}

Chi-square and $t$-tests were used to compare demographic and clinical features between the portion of the population with pulmonary function testing data available, deemed the study population, and the remainder of the registry population to determine whether the study population was representative of the entire population of children presenting to BPD clinic. Linear regression analysis was performed employing both $\mathrm{FEV}_{1} \%$ predicted and $\mathrm{FVC} \%$ predicted as dependent variables and individual predictors as independent variables in univariate models. Predictors were selected based on prior literature. Multivariate regression analysis was performed by dropping non-significant predictors identified as significant in univariate analysis in a stepwise fashion. STATA IC 14 (StatCorp LP, College Station, TX) was used for analyses. $P$-values $<0.05$ were considered statistically significant.

\section{RESULTS}

\section{Demographics and Clinical Characteristics :}

Of the 598 subjects in the registry who were older than five years of age, $88(14.7 \%)$ patients performed adequate pulmonary function testing at a clinic visit between the ages of five and seven years old and thus were included in the study population. The study population was $45.5 \%$ female and had an average gestational age of $26.8 \pm 2.9$ weeks. $63.6 \%$ of subjects identified as non-white race/ethnicity. The mean median household income was $\$ 63,000 \pm \$ 180,800$, and $62.5 \%$ had public insurance. $14.5 \%$ of subjects reported second hand smoke exposure, and $64.5 \%$ reported a family history of asthma. A total of 38 patients $(43.2 \%)$ required supplemental oxygen at the time of discharge from the NICU with an average amount of $0.35 \pm 0.27$ liters per minute. Seventy-eight (88.6\%) patients had data available that allowed them to be classified according to the severity of their BPD with $11.5 \%$ with chronic lung disease of prematurity (no BPD), $7.7 \%$ with mild BPD, $33.3 \%$ with moderate BPD, and $47.4 \%$ with severe BPD. Of the study population, $34.1 \%$ had a gastrostomy tube placed, $22.7 \%$ underwent Nissen fundoplication, and $18.2 \%$ had pulmonary hypertension. In terms of growth parameters, subjects had an average birthweight of $980 \pm 518$ grams with a percentile of $41 \pm 25$ corrected for gestational age. At two years of age, subjects had an average weight percentile for age of $30 \% \pm 31 \%$, an average height percentile for age of $24 \% \pm 26 \%$, and an average BMI percentile for age of $49 \% \pm 31 \%$. The study population had an average $\mathrm{FEV}_{1}$ of $84.5 \%$ predicted $\pm 22.7 \%$ and an average FVC of $94.2 \%$ predicted $\pm 26.5 \%$. See Table 1 for additional demographic characteristics of the study population. Additional analysis illustrated that the study population was not significantly different from the entire registry population in terms of demographic characteristics, clinical characteristics, or growth parameters (Supplemental Table 1 ).

Predictors of Forced Expiratory Volume in 1 second $\left(F E V_{1}\right)$ :

On average, the study population had a $\mathrm{FEV}_{1} \%$ predicted of $84.5 \% \pm 22.7 \%$. Of the demographic characteristics included, in univariate regression analysis, only higher median household income $(2.9+/-1.4, \mathrm{p}=0.044)$ was significantly associated with an improvement in $\mathrm{FEV}_{1} \%$ predicted. There was no significant association between sex $(\mathrm{p}=0.55)$, gestational age $(\mathrm{p}=0.54)$, race/ethnicity $(\mathrm{p}=0.1)$, use of public insurance $(\mathrm{p}=0.36)$, secondhand smoke exposure $(\mathrm{p}=0.74)$, and family history of asthma $(\mathrm{p}=0.99)$ and $\mathrm{FEV}_{1} \%$ predicted at six years of age. The need for supplemental oxygen at the time of NICU discharge $(-11 \pm 5.1, \mathrm{p}=0.035)$ and a greater amount of oxygen required at discharge $(-23.7 \pm 9.9, \mathrm{p}=0.02)$, the presence of gastrostomy tube $(-14.5 \pm 5.2, \mathrm{p}=0.007)$, history of Nissen fundoplication $(-19.6 \pm 5.6, \mathrm{p}=0.001)$, and higher weight percentile at two years of age $(\mathrm{p}=0.026)$ were all associated with significantly lower $\mathrm{FEV}_{1} \%$ predicted at six years of age. However, there was no significant association between a diagnosis of severe BPD $(p=0.051)$, presence of pulmonary hypertension $(\mathrm{p}=0.44)$, personal diagnosis of reactive airway disease or asthma in childhood $(\mathrm{p}=0.61)$, or prescription for inhaled corticosteroid $(\mathrm{p}=0.92)$ and $\mathrm{FEV}_{1} \%$ predicted at age 6 . Weight percen- 
tile at age two was shown to be negatively associated with $\mathrm{FEV}_{1} \%$ predicted at age six $(-0.2 \pm 0.1, \mathrm{p}=0.026)$ but no association was seen between birth weight percentile $(\mathrm{p}=0.63)$, height percentile for age at age two $(\mathrm{p}=0.07)$, or BMI percentile for age at age two $(\mathrm{p}=0.10)$ and $\mathrm{FEV}_{1} \%$ predicted.

Multivariate analysis was performed using the variables found to have association with $\mathrm{FEV}_{1} \%$ predicted in univariate analysis. After adjusting for severity of BPD, a positive association was seen between median household income $(2.6 \pm 1.3, \mathrm{p}=0.04)$ with $\mathrm{FEV}_{1} \%$ predicted whereas a negative association was noted between Nissen fundoplication $(-17.9 \pm 6.2, \mathrm{p}=0.005)$ and weight percentile for age at age $2(-0.2 \pm 0.1$, $\mathrm{p}=0.011)$ and $\mathrm{FEV}_{1} \%$ predicted. Severe $\mathrm{BPD}$ was adjusted for in the model but was not found to be significant $(-5.3 \pm 5.5, \mathrm{p}=0.34)$.

Predictors of Forced Vital Capacity (FVC) :

On average, the study population had a FVC\% predicted of $94.2 \% \pm 26.5 \%$. In univariate regression none of the demographic characteristics were shown to have a significant association with $\mathrm{FVC} \%$ predicted including sex $(p=0.55)$, gestational age $(p=0.54)$, race/ethnicity $(p=0.1)$, median household income $(p=0.25)$, use of public insurance $(\mathrm{p}=0.36)$, secondhand smoke exposure $(\mathrm{p}=0.74)$, and family history of asthma $(\mathrm{p}=0.99)$.

In terms of clinical characteristics, a requirement for supplemental oxygen at the time of discharge from the NICU $(-14 \pm 5.4, \mathrm{p}=0.012)$ and the amount of oxygen required $(-36.7 \pm 10.2, \mathrm{p}=0.001)$ were both found to be negatively associated with FVC\% predicted at six years of age. Additionally, the presence of a gastrostomy tube $(-12.5 \pm 5.7, \mathrm{p}=0.031)$ and Nissen fundoplication $(-19.7 \pm 6.1, \mathrm{p}=0.002)$ were also found to be negatively associated with $\mathrm{FVC} \%$ predicted.

No significant association was seen between FVC\% predicted at six years of age and severity of BPD $(\mathrm{p}=0.08)$, pulmonary hypertension $(\mathrm{p}=0.17)$, personal diagnosis of reactive airway disease or asthma $(\mathrm{p}=0.28)$, or prescription for inhaled corticosteroids $(\mathrm{p}=0.57)$. A significant negative association was found between weight percentile for age at two years of age $(-0.2 \pm 0.1, \mathrm{p}=0.016)$ and $\mathrm{FVC} \%$ predicted at six years of age but no significant association was seen with the other growth parameters analyzed including birth weight percentile $(\mathrm{p}=0.86)$, height percentile for age at age two $(\mathrm{p}=0.11)$, and BMI percentile for age at age two $(\mathrm{p}=0.12)$ and FVC\% predicted.

Multivariate analysis was performed using the variables found in univariate analysis to have an association with FVC\% predicted. The amount of oxygen required at the time of discharge from the NICU $(-26.7 \pm 10.8$, $\mathrm{p}=0.016)$, the presence of Nissen fundoplication $(-14.9 \pm 6.8, \mathrm{p}=0.031)$, and weight percentile for age at age $2(-0.2 \pm 0.1, \mathrm{p}=0.005)$ were found to be negatively associated with FVC\% predicted at six years of age. Severe BPD was adjusted for in the multivariate model but was not found to have a significant association with $\mathrm{FVC} \%$ predicted $(0.2 \pm 6.1, \mathrm{p}=0.98)$.

\section{DISCUSSION}

This study explores several early predictors of pulmonary function via spirometry at six years of age in a group of premature patients with bronchopulmonary dysplasia. Following initial hospital discharge, we found that both the need for supplemental oxygen and the presence of a Nissen fundoplication was associated with lower FVC\% predicted at six years of age. At two years of age, lower median household income and higher weight percentile, but not height percentile, were associated with lower $\mathrm{FEV}_{1} \%$ predicted at six years of age. This study demonstrates that several factors at hospital discharge and at 2 years of age are associated with lung function at 6 years of age. This difference could be attributed to reduced lung growth rates, early-onset or faster decline in lung function, or a combination of both factors.

The traditional model of lung growth and development suggests that lung growth begins in utero and continues through early adulthood, with maximal $\mathrm{FEV}_{1}$ and $\mathrm{FVC}$ values occurring around twenty-five years of age followed by a slow decline throughout the remaining lifespan ${ }^{18}$. For individuals born premature as with term infants, postnatal alveolar "catch up" growth is thought to occur most rapidly in the first two years of life, around the time when most children with bronchopulmonary dysplasia are able to wean from supplemental oxygen ${ }^{13}$. For healthy term individuals who do not smoke and avoid other sources of 
lung damage, normal decline in lung function occurs with age but seldom is associated with significant morbidity or disability. Our data supports previous studies that have reported lower lung function in select groups of individuals, including those with a history of prematurity ${ }^{19-20}$. Indeed, a study from Bui et al. ${ }^{20}$, found that individuals with lower lung function in childhood continued to have lower lung function during adult life. While our results cannot comment on the potential for continued accelerated lung function decline in preterm children with BPD, our findings do suggest that these children are at an increased risk for not achieving optimal peak lung function values in their twenties. This highlights the importance of close pulmonary monitoring and follow-up for preterm patients and especially those with bronchopulmonary dysplasia throughout childhood and into adulthood.

Our data also found that airflow obstruction, represented by a reduced $\mathrm{FEV}_{1} \%$ predicted, was associated with a lower median household income. This finding suggests that children born into lower income households are more likely to have airflow obstruction, similar to an asthma phenotype. This could be attributed to an increase in environmental allergen exposures, potential second-hand smoke exposure, and a more crowded living environment, factors that can adversely affect lung function, particularly in children born premature. Exposure to such environmental insults has been suggested to complicate, and potentially prolong, the resolution of bronchopulmonary dysplasia and prolong risks of pulmonary morbidity ${ }^{21-22}$. Additionally, nonwhite ethnicity was associated with an increased use of public insurance $(\mathrm{p}<0.0001)$ and lower median household income $(\mathrm{p}<0.0001)$, surrogate markers of lower socioeconomic status, suggesting that non-white children with BPD may be disproportionately at risk for lower lung function in part due to their lower socioeconomic status. The presence of a Nissen fundoplication at initial hospital discharge was also associated with a lower $\mathrm{FEV}_{1} \%$ predicted at six years of age. The presence of a Nissen could be a marker for infants with previous swallowing difficulties and microaspiration events; both comorbidities that can exacerbate lung damage and interfere with lung injury repair during a critical period of lung growth.

A diminished lung volume or capacity, represented by decreased FVC\% predicted, was associated with an increase in the amount of supplemental oxygen required at discharge from the NICU. The need for higher supplemental oxygen at initial hospital discharge likely represents that these children had more severe lung injury than those requiring less or no supplemental oxygen. Interestingly, the severity of BPD, as defined by supplemental oxygen requirement at 36 weeks post-menstrual age did not correlate with lung function values at six years of age, indicating that the need for supplemental oxygen or respiratory support at time of discharge is more predictive of lung function at six years of life. Alternatively, children discharged on supplemental oxygen may be more susceptible to post-NICU home environmental exposures, thus preventing adequate postnatal lung catch-up growth. Furthermore, exposure to a higher fraction of inspired oxygen $\left(\mathrm{FiO}_{2}\right)$ in children with BPD may impair alveolar growth during a rapid period of alveologenesis that occurs during the first two years of life ${ }^{13,23}$. In addition to lower $\mathrm{FEV}_{1} \%$ predicted, the presence of a Nissen fundoplication (NF) also predicted a decreased $\mathrm{FVC} \%$ predicted.

Interestingly, a lower weight percentile for age at two years of age was associated with an improved $\mathrm{FEV}_{1} \%$ predicted and an improved FVC\% predicted. While childhood obesity has been associated with a decrease in all lung function parameters ${ }^{24}$, it may not be the etiology of the observed association given the lack of significance of body mass index, a better indicator of obesity in the pediatric population.

\section{Limitations :}

This study was limited by the use of an already recruited study population and clinical information obtained primarily via chart review, some of which may have been missing. Additionally, it was limited by its relatively low sample size $(\mathrm{n}=88)$ due to multiple patients who were lost to follow-up prior to age six. While there was initially concern that the study population could be skewed towards a 'healthier' subset of the registry population given that it includes only patients who are capable of performing pulmonary function testing, the argument was also made that the study population may have captured a 'more affected' population who are still being seen in follow-up in a Pediatric Pulmonary Clinic later in childhood. Overall, the study population does not significantly differ from the registry population on any of the factors included in the analysis (Supplemental Table 1 ). 
There are several other factors that may influence lung function, especially in survivors of preterm birth, that were not accounted for in this study due to its retrospective design including frequent respiratory infections in early childhood which can result in airway inflammation and additional injury in an already compromised lung and other potential damaging environmental insults including exposure to cigarette smoke in-utero, etc.

\section{CONCLUSION}

Our study illustrates that infants who are born premature and develop bronchopulmonary dysplasia have significantly different long-term pulmonary trajectories than what would be expected of "healthy" controls. A decreased $\mathrm{FEV}_{1}$ percent predicted, suggestive of airflow obstruction, was associated with a lower median household income, presence of a Nissen fundoplication, and a lower weight percentile at two years of age whereas a decreased FVC percent predicted, suggestive of decreased pulmonary reserve, was associated with an increased amount of supplemental oxygen required at the time of discharge from the NICU, presence of a Nissen fundoplication, and lower weight percentile at two years of age. Future prospective studies should focus on further clarifying potential modifiable risk factors, particularly those that may be associated with lower household income, that could prevent the development of disease or minimize its impact on later lung function.

\section{REFERENCES}

1. Liu L, Oza S, Hogan D, Perin J, Rudan I, Lawn JE, Cousens S, Mathers C, Black RE. Global, regional, and national causes of child mortality in 2000-13, with projections to inform post-2015 priorities: an updated systematic analysis. Lancet 2015; 385(9966):430-440.

2. Blencowe H, Cousens S, Oestergaard MZ, Chou D, Moller A, Narwal R, Adler A, Garcia CV, Rohde S, Say L, et al. National, regional, and worldwide estimates of preterm birth rates in the year 2010 with time trends since 1990 for selected countries: a systematic analysis and implications. Lancet 2012; 379(9832):2162-2172.

3. Martin JA, Hamilton BE, Osterman MJK. Births in the United States, 2018. NCHS Data Brief 2019; $\operatorname{Jul}(346): 1-8$.

4. Jobe AH, Bancalari E. Bronchopulmonary dysplasia. Am J Respir Crit Care Med 2001; 163(7):17231729 .

5. Collaco JM, McGrath-Morrow SA. Respiratory phenotypes for preterm infants, children, and adults: bronchopulmonary dysplasia and more. Ann Am Thorac Soc 2018; 15(5):530-538.

6. Smith VC, Zupancic JA, McCormick MC, Croen LA, Greene J, Escobar GJ, Richardson DK. Trends in severe bronchopulmonary dysplasia rates between 1994 and 2002. J Pediatr 2005; 146(4):469-473.

7. Kotecha SJ, Edwards MO, Watkins WJ, Henderson AJ, Paranjothy S, Dustan FD, Kotecha, S. Effect of preterm birth on later FEV1: a systematic review and meta-analysis. Thorax 2013; 68(8):760-766.

8. Simpson SJ, Turkovic L, Wilson AC, Verheggen M, Logie KM, Pillow JJ, Hall GL. Lung function trajectories throughout childhood in survivors of very preterm birth: a longitudinal cohort study. Lancet Child Adolesc Health 2018; 2(5):350-359.

9. Fawke J, Lum S, Kirkby J, Hennessy E, Marlow N, Rowell V, Thomas S, Stocks J. Lung function and respiratory symptoms at 11 years in children born extremely preterm: the EPICure study. Am J Respir Crit Care Med 2010; 182(2):237-245.

10. Fortuna M, Carraro S, Temporin E, Berardi M, Zanconato S, Salvadori S, Lago P, Frigo AC, Filippone M, Baraldi E. Mid-childhood lung function in a cohort of children with "new bronchopulmonary dysplasia." Pediatr Pulmonol 2016; 51(10):1057-1064.

11. Von Hove M, Prenzel F, Uhlig HH, Robel-Tillig E. Pulmonary outcome in former preterm, very low birth weight children with bronchopulmonary dysplasia: a case-control follow-up at school age. J Pediatr 2014; 164(1):40-45.

12. Ely DM, Driscoll, AK. Infant mortality in the United States, 2018: data from the period linked birth/infant death file. Natl Vital Stat Rep 2020; 69(7):1-18.

13. Thurlbeck, WM. Postnatal human lung growth. Thorax 1982; 37(8):564-571.

14. Landry JS, Chan T, Lands L, Menzies D. Long-term impact of bronchopulmonary dysplasia on pul- 
monary function. Can Respir J 2011; 18(5):265-270.

15. Oken E, Kleinman KP, Rich-Edwards J, Gillman MW. A nearly continuous measure of birth weight for gestational age using a United States national reference. BMC Pediatr 2003; Jul 8;3:6.

16. Graham BL, Streenbruggen I, Miller MR, Barjaktarevic IZ, Cooper BG, Hall GL, Hallstrand TS, Kaminsky DA, McCarthy K, McCormack MC, et al. Standardization of spirometry 2019 update: an official American Thoracic Society and European Respiratory Society Technical Statement. Am J Respir Crit Care Med 2019; 200(8):e70-88.

17. Cooper BG, Stocks J, Hall GL, Culver B, Steenbruggen I, Carter KW, Thompson BR, Graham BL, Miller MR, Ruppel G, et al. The Global Lung Function Initiative (GLI) Network: bringing the world's respiratory reference values together. Breathe (Sheff) 2017; 13(3):e56-e64.

18. Kerstjens HA, Rijcken B, Schouten JP, Postma DS. Decline of FEV1 by age and smoking status: facts, figures, and fallacies. Thorax 1997; 52(9):820-827.

19. Lum S, Kirkby J, Welsh L, Marlow N, Hennessy E, Stocks J. Nature and severity of lung function abnormalities in extremely pre-term children at 11 years of age. Eur Respir J 2011; 37(5):1199-207.

20. Bui DS, Lodge CJ, Burgess JA, Lowe AJ, Perret J, Bui MQ, Bowatte G, Gurrin L, Johns DP, Thompson $\mathrm{BR}$, et al. Childhood predictors of lung function trajectories and future COPD risk: a prospective cohort study from the first to the sixth decade of life. Lancet Respir Med 2018; 6(7):535-544.

21. Collaco JM, Aherrera AD, Ryan T, McGrath-Morrow SA. Secondhand smoke exposure in preterm infants with bronchopulmonary dysplasia. Pediatr Pulmonol 2014; 49(2):173-178.

22. Doyle LW, Carse E, Adams A, Ranganathan S, Opie G, Cheong JLY. Ventilation in extremely preterm infants and respiratory function at 8 years. N Engl J Med 2017; 377(4): 329-337.

23. McGrath-Morrow SA, Stahl J. Apoptosis in neonatal murine lung exposed to hyperoxia. Am J Respir Cell Mol Biol 2001; 25(2):150-155.

24. Forno E, Han YY, Mullen J, Celedón JC. Overweight, Obesity, and Lung Function in Children and Adults-A Meta-analysis. J Allergy Clin Immunol Pract 2018; 6(2):570-581.

\section{Hosted file}

Tables.pdf available at https://authorea.com/users/366018/articles/485913-predictors-ofpulmonary-function-at-six-years-of-age-in-infants-with-bronchopulmonary-dysplasia 